\title{
Analyse des avantages et des coûts de la politique de bas prix de l'énergie hydroélectrique : le cas d'Alcan au Saguenay-Lac-Saint-Jean
}

\author{
Gilles Bergeron ${ }^{1}$ \\ Université du Québec à Chicoutimi
}

$\Gamma^{\prime}$

utilisation des ressources hydroélectriques est un enjeu important pour le développement du Québec, et plus particulièrement pour le développement des régions ressources.

Faut-il maintenir une politique de bas prix de l'énergie pour favoriser la création ou encore le maintien des emplois dans les entreprises énergivores compte tenu de leur contribution à la création d'emplois et au développement économique, ou encore faut-il augmenter les prix et les possibles exportations d'énergie pour augmenter les revenus d'Hydro-Québec et du gouvernement du Québec à des fins de développement économique et social à préciser ?

Le calcul des avantages et des coûts d'une politique de bas prix de l'énergie et leur évolution sur une période de 23 ans pourrait nous aider à répondre à cette question.

Du point de vue des régions ressources qui bénéficient particulièrement de cette stratégie des bas prix de l'énergie, tout changement ne pourra être envisagé et devenir acceptable que dans la mesure où une partie des gains d'une nouvelle stratégie soit utilisée pour compenser les pertes subies dans les régions ressources et pour mettre en œuvre une politique de reconversion du développement de ces régions.

Notre article porte sur les avantages et les coûts d'une politique de bas prix de l'énergie pour Alcan dans la région du Saguenay-Lac-Saint-Jean et ce, en tenant compte des conditions particulières d'Alcan à titre de producteur privé.

\section{Les avantages d'une politique de bas prix de l'énergie mesurés par la contribution d'Alcan au développement de la région en 1980 et en 2003}

Le tableau 1 de la page suivante permet de mesurer l'évolution de la contribution d'Alcan au développement de la région de même que les changements dans les principaux paramètres de la production. Nous avons choisi ces deux années de référence compte tenu de la qualité des informations disponibles ainsi que du caractère représentatif de ces deux années. Pour tenir compte des effets de l'inflation dans la comparaison des données monétaires, nous avons calculé les données de 1980 en dollars de 2003 à l'aide de la feuille de calcul de l'inflation de la Banque du Canada.

De 1980 à 2003, le nombre d'emplois directs chez Alcan dans la région est passé de 9270 à 6115 , soit une diminution de $34 \%$. Malgré cette situation, la masse salariale en dollars de 2003 a augmenté de 547,4 à 598 millions de dollars, soit une hausse de $9 \%$. Les augmentations de salaires ont plus que compensé les pertes d'emplois, ce qui a un impact favorable sur les emplois induits qui dépendent du pouvoir d'achat des travailleurs d'Alcan. En dollars de 2003, le salaire moyen incluant les bénéfices marginaux a augmenté de $68 \%$, ce qui est une augmentation importante. Elle s'explique en partie par l'augmentation de la productivité des travailleurs.

Les achats de biens et services dans la région en dollars de 2003 augmentent de 263 à 295 millions durant 
la même période, soit de $12 \%$. Ce résultat est faible et semble surprenant compte tenu de l'augmentation de la production et des efforts déployés par Alcan.

Les investissements courants (qui représentent les investissements annuels consentis pour maintenir les installations en état de produire) augmentent de 92 à 111 millions en dollars de 2003, soit de $20 \%$. Ce résultat s'explique par l'augmentation de la valeur des installations.
La production d'aluminium dans la région passe de 510,9 à 977,8 mille tonnes métriques, soit une augmentation très considérable de $91 \%$. Chaque travailleur produisait 55,11 tonnes métriques par an en 1980, alors qu'il en produit 159,3 en 2003. La production par travailleur augmente de $189 \%$. Ces changements majeurs s'expliquent largement par l'introduction de technologies nouvelles résultant d'un programme d'investissement de 7 milliards de dollars durant cette période.

\section{Tableau 1 - Évaluation de la contribution des opérations d'Alcan au développement de la région du Saguenay-Lac-Saint-Jean}

\begin{tabular}{|c|c|c|c|c|}
\hline & 1980 & $1980 \$ 2003)$ & 2003 & \% chang. \\
\hline $\begin{array}{l}\text { Nombre d'emplois } \\
\text { Alcan }\end{array}$ & $9270^{(1)}$ & & $6115^{(3)}$ & $-34 \%$ \\
\hline $\begin{array}{l}\text { Masse salariale (Alcan) } \\
\text { (millions) }\end{array}$ & $226,6^{(1)}$ & 547,4 & $598^{(3)}$ & $+9 \%$ \\
\hline $\begin{array}{l}\text { Achat de B et S } \\
\text { (millions) }\end{array}$ & $108,6^{(1)}$ & 263 & $295^{(3)}$ & $+12 \%$ \\
\hline $\begin{array}{l}\text { Investissements } \\
\text { courants (millions) }\end{array}$ & $38^{(1)}$ & 92 & $111^{(3)}$ & $+20 \%$ \\
\hline $\begin{array}{l}\text { Production d'aluminium } \\
\text { primaire en tonnes } \\
\text { métriques (1000) }\end{array}$ & $510,9^{(2)}$ & - & $977,8^{(3)}$ & $+91 \%$ \\
\hline $\begin{array}{l}\text { Production d’énergie } \\
\text { d'Alcan dans la région }\end{array}$ & $1812 \mathrm{MW}^{(2)}$ & - & $2000 \mathrm{MW}^{(3)}$ & $+10 \%$ \\
\hline $\begin{array}{l}\text { Consommation d’énergie } \\
\text { production régionale } \\
\text { Alcan }\end{array}$ & $1129 \mathrm{MW}^{(4)}$ & - & $1635 \mathrm{MW}^{(4)}$ & $+45 \%$ \\
\hline $\begin{array}{l}\text { Valeur ajoutée } \\
\text { régionale (millions) }\end{array}$ & $364,7^{(1)}$ & 881,6 & $983^{(4)}$ & $+11 \%$ \\
\hline $\begin{array}{l}\text { Emplois totaux } \\
\text { (multiplicateur 1.6) }\end{array}$ & $14755^{(1)}$ & - & $9781^{(4)}$ & $-34 \%$ \\
\hline $\begin{array}{l}\text { (1) Données NAPI }{ }^{2} \\
\text { (2) } \text { Données Alcan }^{3}\end{array}$ & $\begin{array}{l}\text { (3) Donné } \\
\text { (4) Estime }\end{array}$ & $\begin{array}{l}\text { an }^{4} \\
\text { Bergeron }\end{array}$ & & \\
\hline
\end{tabular}

La production d'énergie par Alcan augmente de $1812 \mathrm{MW}$ à $2000 \mathrm{MW}$ par an, soit de $10 \%$ selon les données fournies par Alcan. La consommation d'énergie pour la production d'Alcan dans la région augmente de $1129 \mathrm{MW}$ à $1635 \mathrm{MW}$, soit de $45 \%$. C'est une augmentation moins forte que l'augmentation de la production du fait que les nouveaux processus de production sont moins énergivores de $27 \%$. La consommation d'énergie par travailleur augmente de
119 \%. Nous avons estimé la consommation d’énergie en tenant compte de la production d'aluminium primaire réalisée dans la région et de la consommation d'énergie selon les paramètres techniques des usines. Nous avons ajouté $100 \mathrm{MW}$ pour tenir compte des pertes et autres besoins de l'entreprise. En tenant compte des deux usines de la Mauricie, la consommation d'énergie est de $1297 \mathrm{MW}$ en 1980 et de 1956 MW en 2003. 
La valeur ajoutée régionale augmente de 881,6 millions à 983 millions de dollars, soit de $11 \%$, alors que les emplois totaux dans la région liés aux activités d'Alcan, soit les emplois directs, indirects et induits, passent de 14755 emplois en 1980 à 9781 en 2003, soit une diminution de $34 \%$.

\section{Les avantages de producteur privé se mesurent par la différence entre le coût de production de l'énergie pour Alcan et le tarif L pour $2000 \mathrm{MW}$.}

\section{Des avantages qui diminuent en termes d'emplois}

En résumé, sur une période de 23 ans, la compagnie a augmenté sa production de façon significative, soit de $91 \%$. Cette augmentation de la production s'est accompagnée d'une augmentation moindre de la consommation d'énergie, soit de $45 \%$, et d'une augmentation plus grande de la productivité, soit de $189 \%$, ce qui a provoqué une baisse des emplois directs de $34 \%$. L'augmentation en termes réels de la masse salariale de $9 \%$, l'augmentation des achats de biens et services de $12 \%$, l'augmentation des investissements courants de $20 \%$ ont permis une augmentation de la valeur ajoutée régionale de $11 \%$. Les emplois totaux dans la région ont cependant diminué de $34 \%$, soit une baisse de 4974 emplois malgré une augmentation de 91 \% de la production d’aluminium.

\section{Les coûts d'une politique de bas prix de l'énergie ou les avantages concédés à Alcan}

\section{Les coûts en 1980}

Les coûts d'une politique de bas prix de l'énergie sont relativement faibles en 1980 compte tenu que le Québec dispose d'énergie produite à bas coût et que cette énergie ne peut être exportée sur les marchés américains en raison des technologies disponibles et des contraintes réglementaires.

Pour chiffrer les avantages concédés à Alcan, nous avons calculé les avantages de producteur privé pour les $1812 \mathrm{MW}$ produits par Alcan, soit la différence entre le coût de production d'Alcan estimé à 0,225\$/ MWh et le tarif L 1,47థ/MWh. Le coût est estimé à
193,6 millions en dollars de 1980 et à 468,9 millions en dollars de 2003.

\section{Les coûts en 2003}

Pour calculer les coûts d'une politique de bas prix de l'énergie dans une perspective régionale, nous avons effectué le calcul en deux étapes pour distinguer les avantages dont Alcan profite à titre de grande entreprise, au même titre que les autres grandes entreprises du Québec, et les avantages particuliers dont elle profite à titre de producteur privé.

Les avantages à titre de grande entreprise se mesurent par la différence entre le tarif $L$ et le prix sur le marché à l'exportation, soit le coût d'opportunité de 4,3థ pour les 1635 MW consommés pour la production régionale. Nous obtenons un avantage de 616 millions de dollars par an en 2003. Un calcul en utilisant la méthode des coûts économisés donnerait des résultats comparables.

Les avantages de producteur privé se mesurent par la différence entre le coût de production de l'énergie pour Alcan et le tarif L pour $2000 \mathrm{MW}$, soit la production déclarée par Alcan. En utilisant le coût de production de $0,9 \$ / K W h$, soit le coût de production selon le syndicat, nous obtenons un avantage de 490 millions, alors qu'en utilisant un coût de 1,59థ/KWh estimé à partir des coûts historiques d'Hydro-Québec, nous obtenons 369 millions.

\section{Des coûts qui doublent}

Le coût de la politique de bas prix de l'énergie estimé à partir des avantages concédés à Alcan dans la région se chiffre entre 1 et 1,1 milliard de dollars par an en 2003, soit une augmentation de $113 \%$ ou de $134 \%$ par rapport à 1980. Cette augmentation s'explique à la fois par l'augmentation du prix de l'énergie et par les progrès technologiques qui font augmenter la consommation d'énergie par travailleur.

\section{En conclusion}

Les coûts d'une politique de bas prix de l'énergie hydroélectrique et leur évolution dans le temps sont directement liés aux progrès technologiques de même qu'au prix de l'énergie. Il est évident qu'au démarrage de l'industrie de l'aluminium au Québec et dans la 
région du Saguenay-Lac-Saint-Jean, le coût de production de l'énergie hydroélectrique était très bas et les autres clients peu nombreux compte tenu des technologies disponibles pour le transport de l'énergie et de la réglementation en vigueur. L'aluminium était un moyen approprié et efficace pour mettre en valeur et exporter notre énergie. À mesure que le coût de production de l'énergie augmente et que les autres clients sont disposés à payer un prix plus élevé, les coûts d'une politique de bas prix de l'énergie augmentent de façon significative, comme le démontrent nos calculs pour l'année 1980 en comparaison avec l'année 2003.

Selon notre étude, sur une période de 23 ans, les gains de la politique de bas prix de l'énergie augmentent de $11 \%$ en termes de valeur ajoutée régionale, mais diminuent de $34 \%$ en termes d'emplois, alors que les coûts sur la même période de cette politique font plus que doubler. Dans les circonstances, on peut douter de la sagesse et de l'opportunité de poursuivre la même politique pendant les 25 prochaines années sans apporter des correctifs majeurs. Des changements importants vont survenir. Les régions ressources doivent développer des stratégies pour défendre leurs intérêts dans ce nouveau contexte. Elles doivent chercher à la fois à profiter des augmentations de prix par le biais de redevances régionales et des nouveaux revenus tirés des surplus d'énergie dégagés à l'occasion de la fermeture d'usines.

Alcan annonce d'ailleurs qu'elle a l'intention de poursuivre la fermeture de ses vieilles usines au Québec au cours des prochaines années sans entreprendre de projets majeurs de construction. Le surplus d'énergie dégagé dans la région du Saguenay-Lac-SaintJean serait de $700 \mathrm{MW}$ pour une valeur économique estimée à 373 millions en dollars de 2003.

\section{On peut douter de la sagesse et de l'opportunité de poursuivre la même politique pendant les $\mathbf{2 5}$ prochaines années sans apporter des correctifs majeurs.}

C'est un enjeu économique majeur. Une stratégie cohérente et solidaire et une mobilisation régionale sont nécessaires pour éviter que le partage soit négocié entre Hydro-Québec et Alcan. À titre de propriétaire d'Hydro-Québec, le Gouvernement du Québec est en conflit d'intérêt et, quel que soit le parti au pouvoir, ne peut défendre les intérêts de la région dans ce dossier.

La Conférence régionale des élus (CRÉ) doit former un comité technique et examiner divers scénarios pour maximiser les retombées régionales de la production d'énergie et pour s'assurer que, si Alcan n'est pas en mesure d'utiliser toute l'énergie dont elle dispose, les revenus tirés de ce surplus reviennent à la collectivité régionale.

Le Syndicat des TCA a soumis une hypothèse de travail intéressante avec sa proposition d'énergie patrimoniale. D'autres hypothèses complémentaires devraient également être envisagées pour s'assurer de maximiser les retombées régionales en termes d'emplois.

\section{Si Alcan n'est pas en mesure d'utiliser toute l'énergie dont elle dispose, les revenus tirés de ce surplus reviennent à la collectivité régionale.}

La passivité avec laquelle les leaders régionaux ont accepté qu'Hydro-Québec augmente ses profits de 56 millions de dollars par an à l'occasion de la fermeture des cuves Söderberg, alors que la région perdait 550 emplois permanents, nous laisse cependant perplexe pour l'avenir.

\section{Notes et références}

1 Gilles Bergeron est économiste et professeur à l'Université du Québec à Chicoutimi. Il et directeur du département des sciences économiques et administratives de cette institution.

2 Napi, C., L. Courville, M. Dagenais, P. Hubert et H. Taguwaï, L'impact économique de l'Alcan au Québec et au Lac-Saint-Jean, rapport de recherche.

3 Alcan, Rapport annuel aux employés 1984.

$4 \quad$ Alcan au Québec, Guide de presse 2004. 